Revue des patrimoines

\title{
Les maisons à avancée en Bretagne entre 1600 et 1900 : essai de chronologie pour un type emblématique
}

\section{Christel Douard}

\section{OpenEdition}

Journals

Édition électronique

URL : http://journals.openedition.org/insitu/3467

DOI : 10.4000/insitu.3467

ISSN : 1630-7305

Éditeur

Ministère de la culture

Référence électronique

Christel Douard, «Les maisons à avancée en Bretagne entre 1600 et 1900 : essai de chronologie pour un type emblématique », In Situ [En ligne], 8 | 2007, mis en ligne le 01 mars 2007, consulté le 30 avril 2019. URL : http://journals.openedition.org/insitu/3467 ; DOI : 10.4000/insitu.3467

Ce document a été généré automatiquement le 30 avril 2019.

\section{(c)}

In Situ Revues des patrimoines est mis à disposition selon les termes de la licence Creative Commons Attribution - Pas d'Utilisation Commerciale - Pas de Modification 4.0 International. 


\title{
Les maisons à avancée en Bretagne entre 1600 et 1900 : essai de chronologie pour un type emblématique
}

\author{
Christel Douard
}

1 De plan rectangulaire comme la plupart des logis ruraux de la région, la maison à avancée - elle existe dans une vaste aire géographique de la Bretagne sur une très longue période se distingue par un, voire plusieurs avant-corps latéraux. Le ou les avant-corps peuvent avoir des profondeurs et des fonctions variables. Placée en prolongement du pignon, la partie saillante, généralement située sur l'élévation principale, plus rarement sur l'élévation postérieure, prend, au cours du temps, des formes et des proportions diverses ${ }^{1}$ (fig. $\left.n^{\circ} 1, n^{\circ} 2\right)$. 


\section{Figure 1}

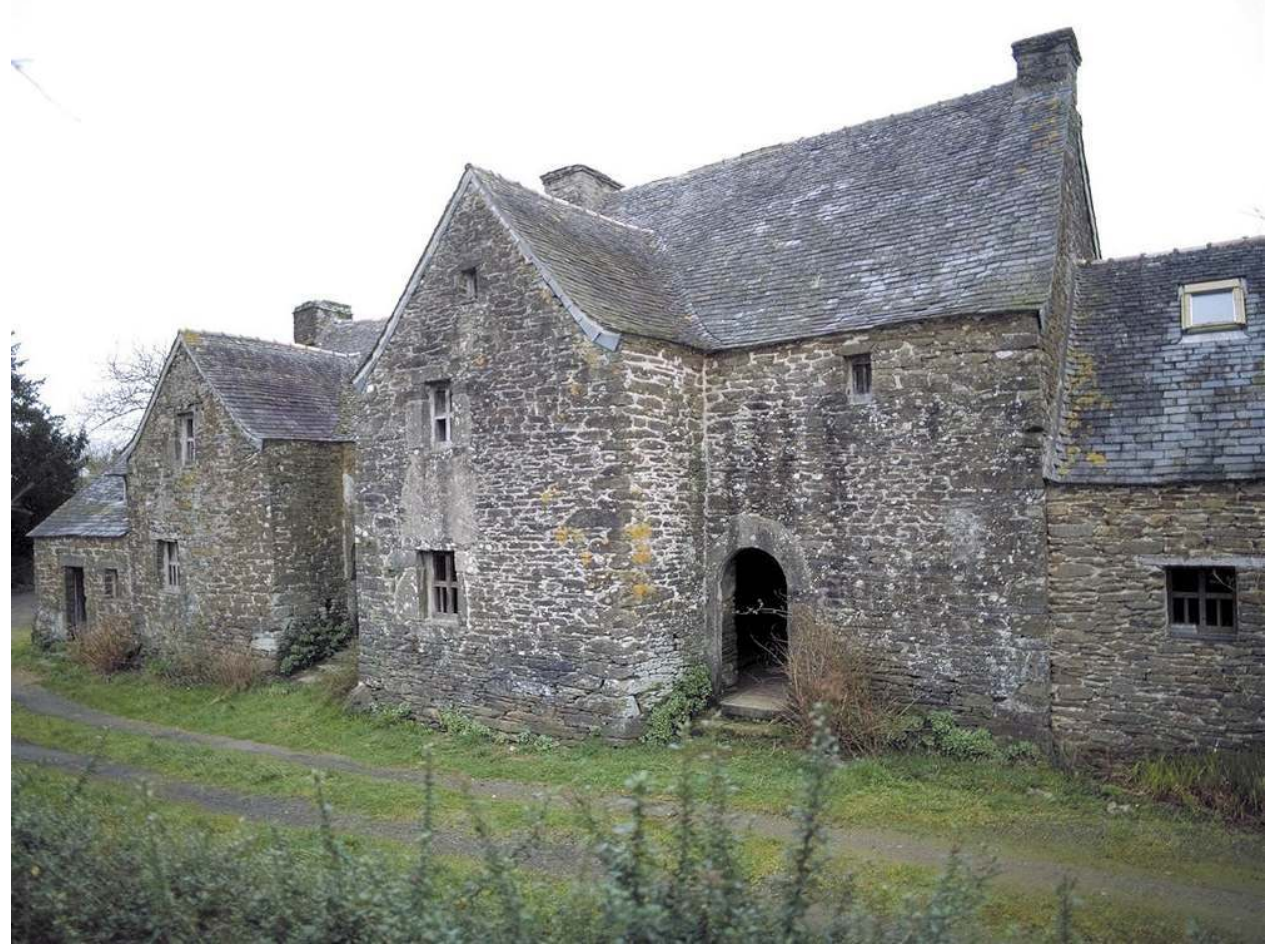

Brasparts, Botbern. Logis jumelés datés respectivement 1696 (à droite) et 1725 (à gauche). Bègne, Bernard

(c) Inventaire général, ADAGP, 1999. 
Figure 2

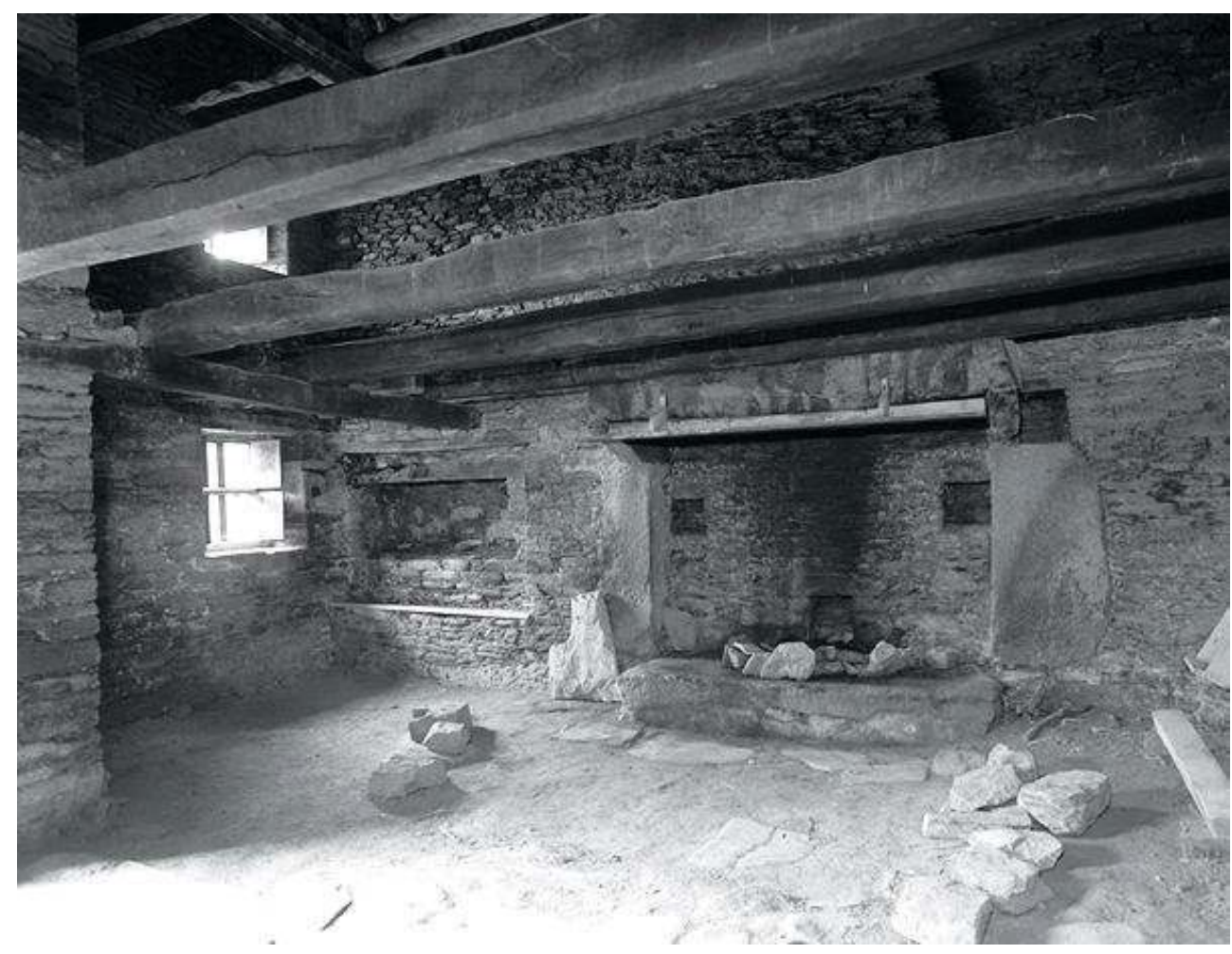

Brasparts, Botbern. Logis daté 1696. Pièce commune. Bègne, Bernard

(c) Inventaire général, ADAGP, 1999.

\section{Aire de diffusion}

2 Les maisons à avancée font partie de la catégorie des logis sans cohabitation avec le bétail ; elles sont caractéristiques de la partie nord-ouest de la Bretagne, dans une zone qui comprend le Léon, le nord de la Cornouaille et l'ouest du Trégor (fig. $\mathbf{n}^{\circ} 3$ ). 


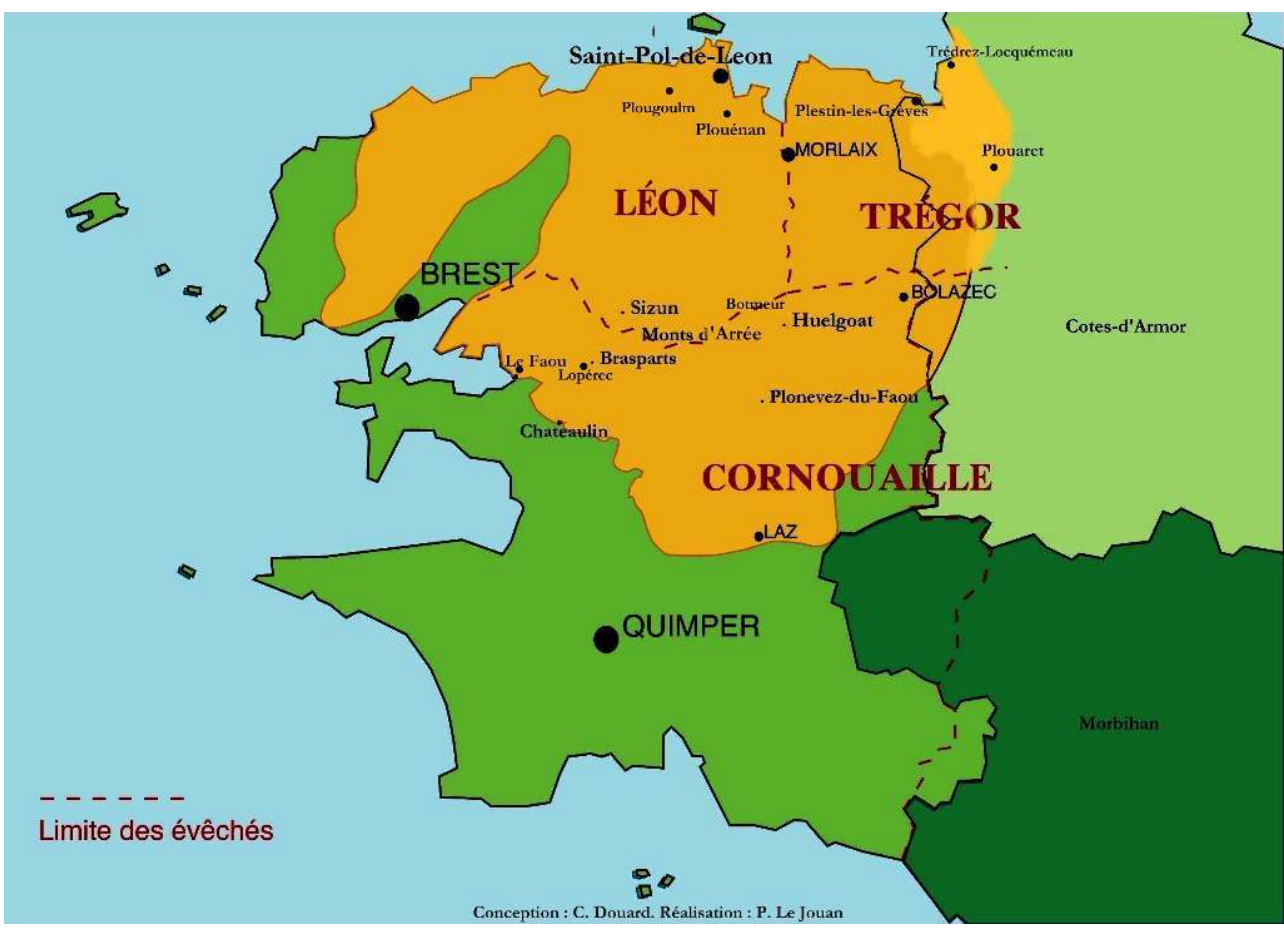

Carte : aire de diffusion des maisons à avancée (Conception : C. Douard. Réalisation : Pierrick Le Jouan, centre départemental de la documentation pédagogique, Brest)

(c) Inventaire général, ADAGP, 2000

La carte montre que les franges du littoral du bas Léon et les îles en sont dépourvues. En descendant vers le sud, le phénomène s'observe entre Plougastel-Daoulas, Édern et Laz ; il ne dépasse pas l'ouest de Châteaulin, mais franchit largement la vallée de l'Aulne retenue indûment comme limite $\mathrm{sud}^{2}$. Les frontières entre les évêchés de l'Ancien Régime indiquent que le secteur anciennement rattaché à l'évêché de Tréguier et aujourd'hui situé à cheval sur les départements du Finistère et des Côtes-d'Armor est largement concerné, même si le type y devient progressivement marginal ${ }^{3}$ (fig. $\mathbf{n}^{\circ} 4$ ). 


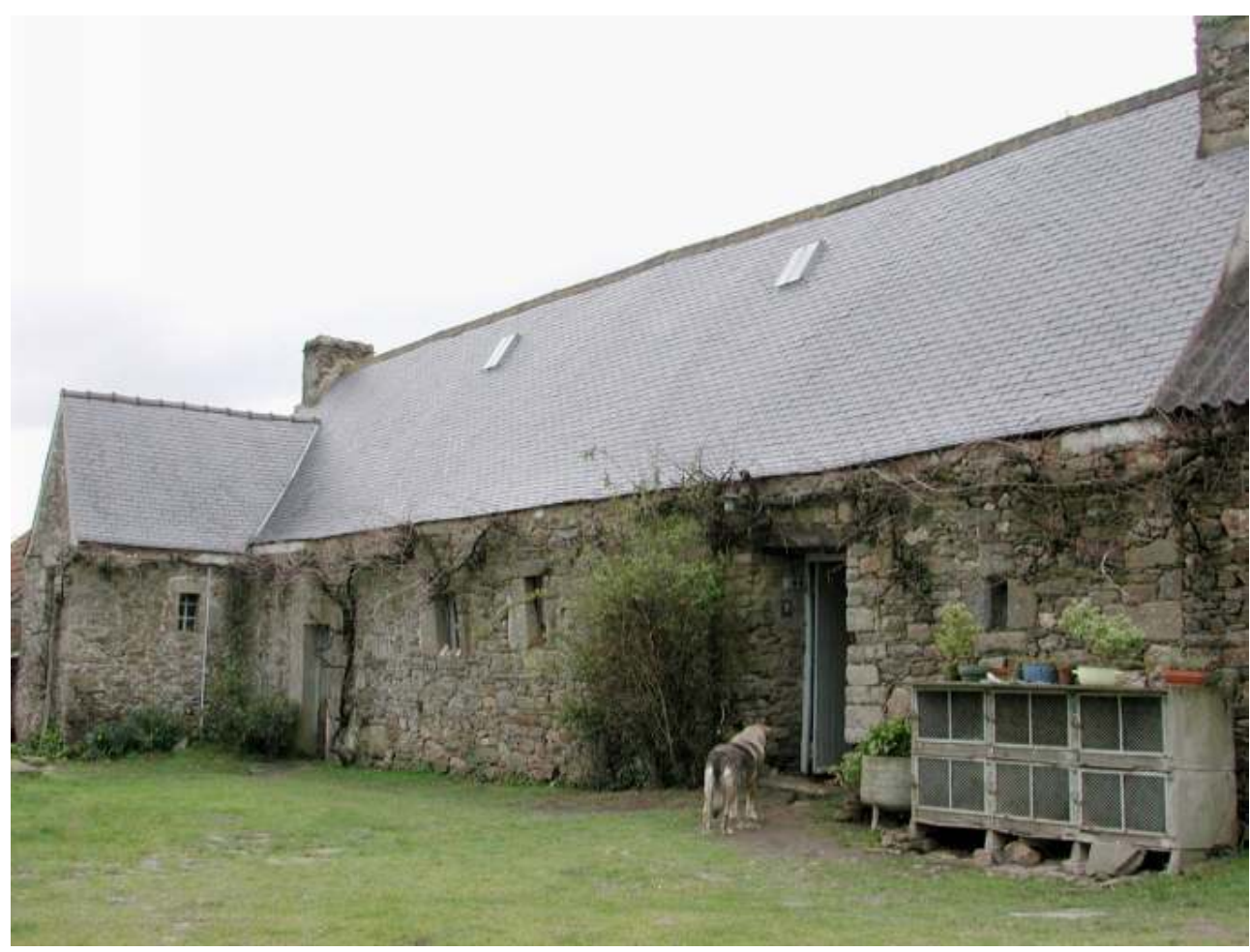

Tréduder, Le Veuzit. Logis de ferme (XVIIe siècle) avec petite avancée appelée « kuz tol » (cache-table). Pichouron, $P$.

(C) Inventaire général, 2004, (c) Conseil général des Côtes-d'Armor, 2004.

4 Les densités sont très variables. Les fréquences s'avèrent plus faibles dans les zones périphériques, notamment dans le bas Léon ou encore autour de Callac et Plouaret. Dans la région de Saint-Pol-de-Léon, $25 \%$ des maisons rurales recensées en 1985 par le service régional de l'Inventaire étaient pourvues d'avancées, avec une proportion particulièrement élevée autour de l'ancienne ville épiscopale. Dans les environs du Faou, le type à avancée représentait $12 \%$ de la totalité des maisons répertoriées en $1996^{4}$. Environ $30 \%$ des 200 logis répertoriés en 2006 sur la seule commune de Sizun possèdent une ou plusieurs avancées ${ }^{5}$.

\section{Le nom et la chose}

5 Aux différents secteurs géographiques correspondent plusieurs termes en breton pour désigner l'avancée.

6 Jean Lagadeuc, dans le Catholicon ${ }^{6}$, dictionnaire breton-français-latin paru en 1499, propose le mot appenteice pour traduire "appentis de maison", terme neutre qui ne recouvre pas nécessairement cette structure particulière. En l'absence de maisons conservées de cette époque, il est donc difficile de savoir si le terme désigne des maisons de ce type construites plus tardivement, globalement entre le XVIIe siècle et le début du XXe siècle; les témoins les plus anciens conservés ne semblent pas être antérieurs au début du XVIIe siècle (fig. $\mathbf{n}^{\circ}$ 5). 


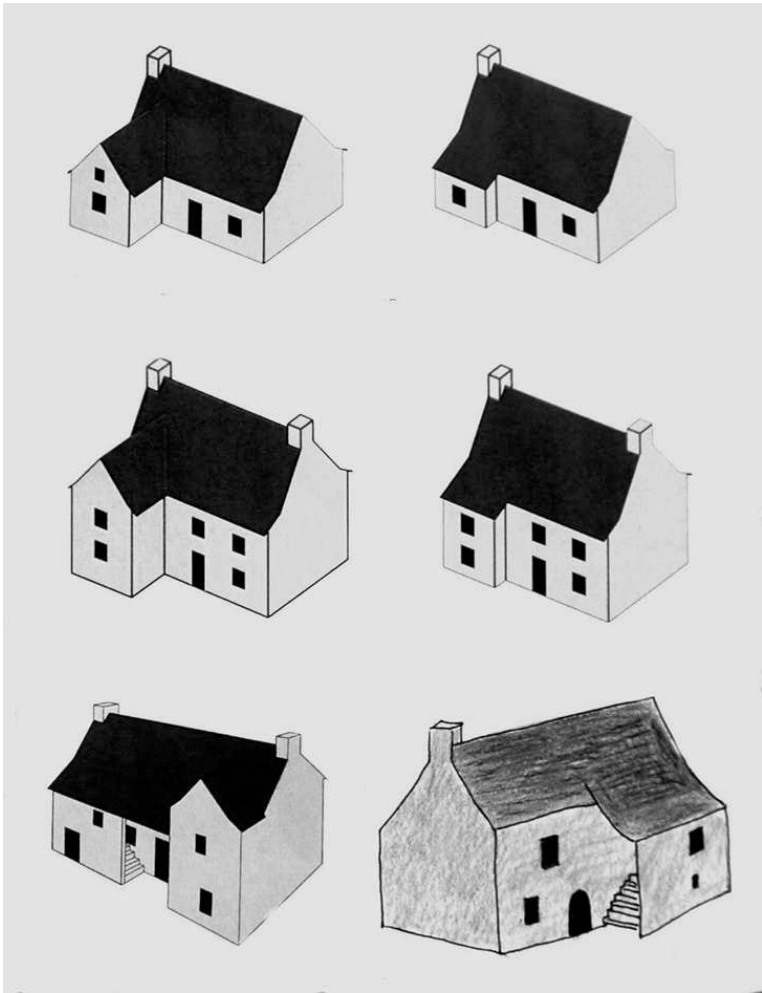

Schéma des variantes dominantes (Conception : C. Douard. Réalisation : Pierrick Le Jouan, centre départemental de la documentation pédagogique, Brest)

(c) Inventaire général, ADAGP, 2000.

En 1732, Grégoire de Rostrenen, dans son Dictionnaire François-celtique ou François-breton ${ }^{7}$ donne les mots appantéiz, apateiz, appenteiz ou appoteiz pour le «toit qui n'a de pente que d'un côté ». A cette période, le type, avec un grand nombre de variantes, est largement répandu dans la zone indiquée.

Apothéis (qui devient localement «à poteis" - on entend aussi que la maison «a un potéis ») n'est donc ni plus ni moins qu'une déformation du mot français "appentis », alors que dans la zone étudiée, architecturalement, il s'agit bien d'un avant-corps et non d'un appentis.

Essentiellement en Cornouaille, dans les monts d'Arrée et dans le sud du haut Léon, le terme de apoteiz est toujours en usage. Dans la plus grande partie du haut Léon, on dit apothis taol (avancée de la table). De même, dans le bas Léon, on désigne cette caractéristique architecturale, souvent de structure semi-circulaire, comme avans taol. En Trégor finistérien, entre Plougasnou et Botsorhel, tout comme dans la région de Plestinles-Grèves et Plouaret, c'est le terme kuz tol (cache table) qui est utilisé.

Il apparaît clairement que dans le nord et l'est de la zone, l'avant-corps, comme les termes bretons d'ailleurs le précisent, sert exclusivement à placer la table (et les bancs). En revanche, dans le sud, l'avancée est désignée par un terme plus générique (apoteiz, appentis) : la partie en saillie peut abriter le mobilier, ce qui est majoritairement le cas, mais, en plus, l'escalier de distribution extérieur en maçonnerie, phénomène très fréquemment observé dans la zone des monts d'Arrée alors qu'il est exceptionnel, voire inexistant, dans le nord du Léon et le Trégor (fig. $\mathbf{n}^{\circ}$ 6). 
Figure 6

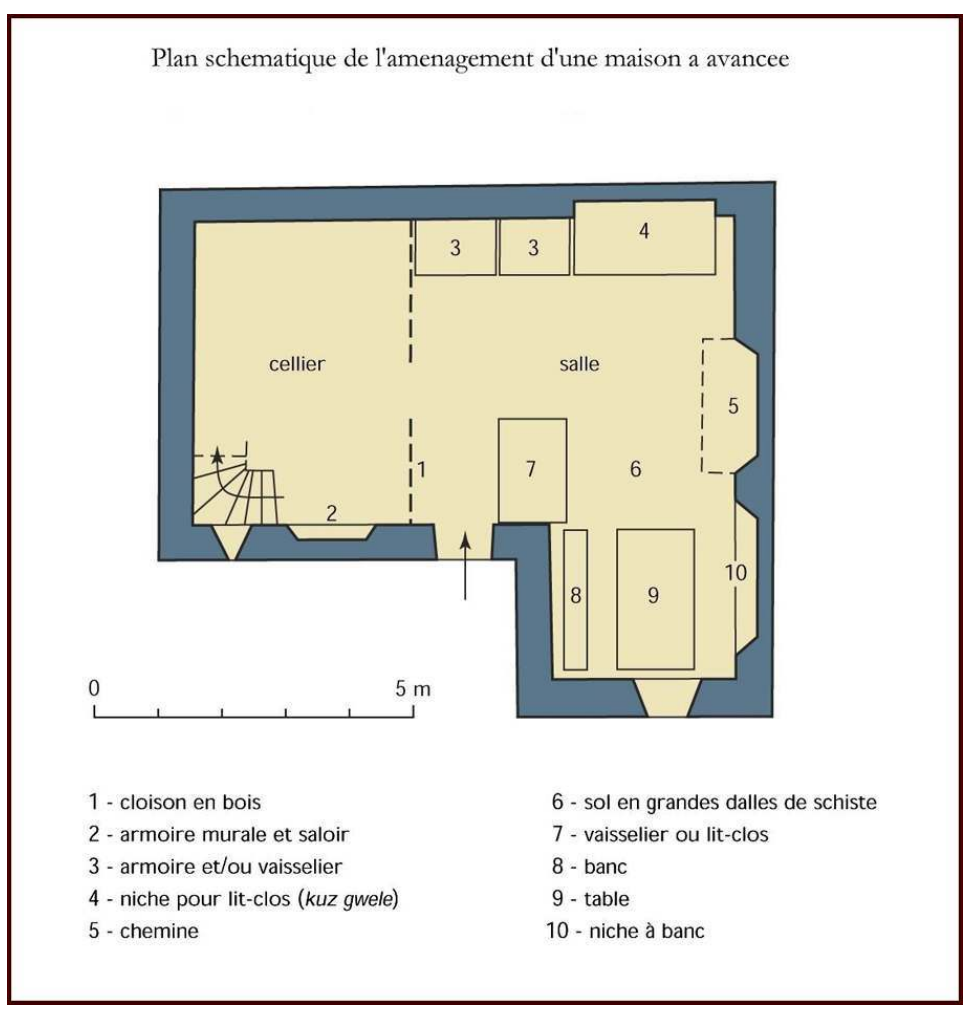

Plan schématique de l'aménagement traditionnel d'une maison à avancée (Conception : C. Douard Réalisation : Pierrick Le Jouan, centre départemental de la documentation pédagogique, Brest)

(c) Inventaire général, ADAGP, 2000.

Dans les actes notariés, rédigés en français et sans référence aux termes bretons, cette particularité architecturale est parfois mentionnée avec précision. Ainsi en 1657 autoriset-on François Guillerm, laboureur demeurant au village de Kervern en Pleyber-Christ, de "bastir un recoign ou bouttehor qui aura treize pieds de largeur par dehors et sept de longeur [4,30 $\mathrm{m}$ de large, 2,30 $\mathrm{m}$ en avancée] qui sera de la mesme hauteur de muraille que la muraille de la maison $»^{8}$. Dans d'autres documents plus tardifs mais relatifs aux maisons du même village, des termes comme cache table (1716), bout dehors (1750), bout de dehors (1771) ou saillie (1791) sont utilisés, ce qui laisse supposer un usage courant. A Lopérec, on décrit, dans un procès-verbal de 1806, une maison construite au XVIIe siècle avec son « avancé en dehors de soixante cinq centimettres »".

\section{Paysans, journaliers, marchands et prêtres}

La diversité du rang social des commanditaires - toute la population rurale, à l'exception de la noblesse, est concernée - montre à quel point cette forme d'habitat a fini par s'imposer durablement. Le ou les modèles ne sont donc pas réservés à un groupe précis comme une idée reçue et indûment répandue voulait le faire croire ${ }^{10}$. En effet, il convient de mettre en doute l'affirmation suivant laquelle la maison à avancée correspondrait exclusivement à l'habitat d'artisans tisserands ou de marchands de toiles. L'artisanat du lin (blanchiment des toiles, tissage) était, certes, largement répandu dans une grande zone du secteur concerné, mais cette économie domestique ne définissait pas une forme 
particulière d'habitat. Le type, on le voit, est répandu au-delà des zones de transformation et de tissage du lin et l'avancée n'était pas forcément réservée à l'emplacement d'un métier à tisser. D'autre part, le nombre élevé de constructions réalisées pour des prêtres, des laboureurs ou des journaliers - constructions de dimensions tantôt importantes, tantôt modestes - met non seulement à mal cette attribution limitative mais prouve plutôt le contraire : la forme architecturale du logis n'est pas déterminée par l'activité économique ou le métier du commanditaire mais correspond à une manière de faire localement enracinée, une " mode » adaptée par tous ceux vivant à la campagne à un moment donné. Il s'agit bien davantage d'une acceptation tacite et généralisée, d'une "expression d'un assentiment général qui aboutit à un système répétitif des traits majeurs de la construction $»^{11}$ (fig. $\left.\mathbf{n}^{\circ} 7, \mathbf{n}^{\circ} \mathbf{8}\right)$.

Figure 7

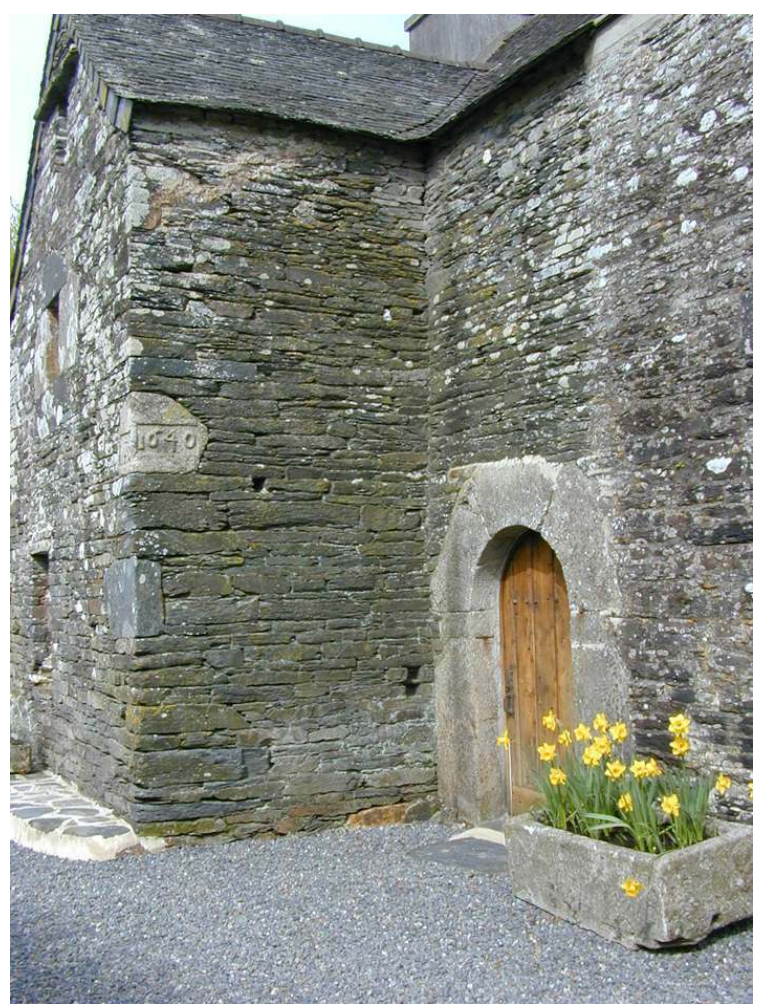

Sizun, Saint-Cadou Kergudon. Logis, peut-être d'un prêtre, daté 1640. Douard, Christel (c) Inventaire général, ADAGP, 2006. 
Figure 8

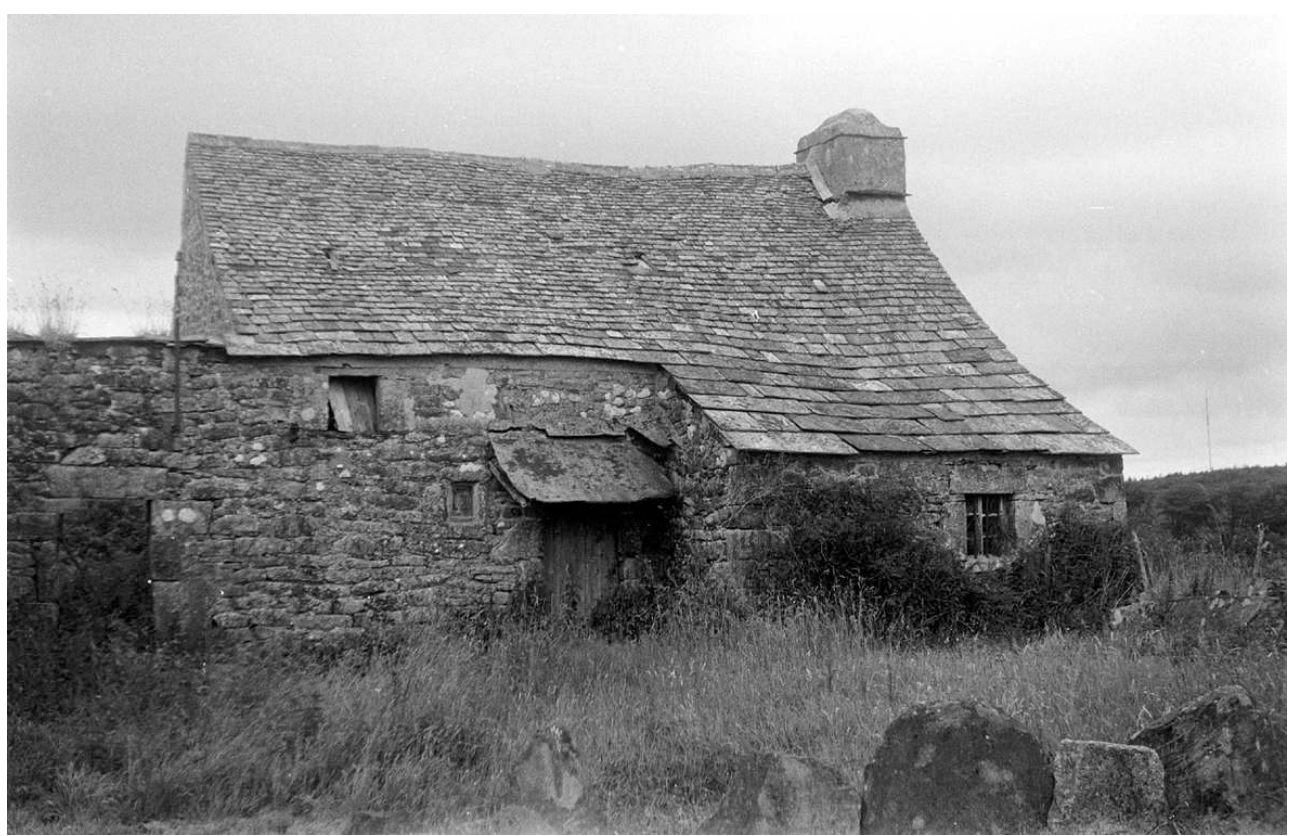

Botmeur, Sallou. Ancien logis de ferme (début XIXe siècle ?), état en 1969.

(c) Inventaire général, ADAGP, 2006.

13 Les maisons les plus soignées qui portent souvent un chronogramme, témoignent de l'aisance de leurs commanditaires, en majorité paysans, marchands ou prêtres. Mais le type n'est pas réservé aux seuls notables ruraux: s'agissant aussi d'un mode de construction, la population moins aisée s'y réfère en bâtissant des édifices de volumes plus modestes et souvent sans étage. 


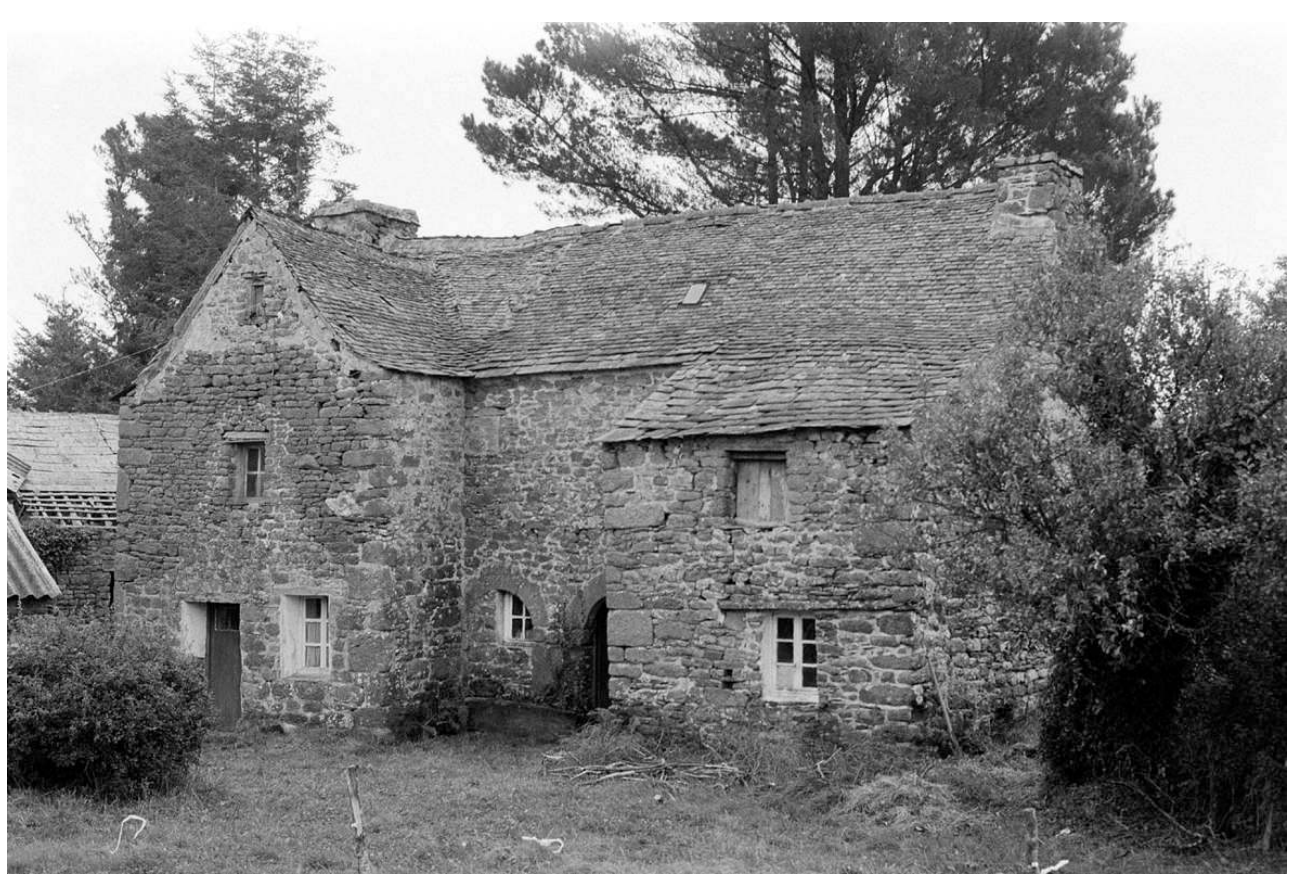

Sizun, Kerambloch. Le bâtiment, avec deux avancées dont une, à droite, abrite l'escalier, a été construit en 1665. La présence d'un mur de refend (disparu) induit qu'il s'agissait, à l'origine, de deux logis jumelés. Etat en 1970. Pape, L.

(c) Inventaire général, ADAGP, 2006.

Les bourgs n'en sont pas dépourvus, à l'instar de celui de Sizun qui, au début du XIXe siècle, comptait une trentaine de maisons à avancée associées à un escalier extérieur de distribution ; une seule, à l'état de vestiges, subsistait en 2006 (voir fig. $\mathbf{n}^{\circ}$ 13) (fig. $\mathbf{n}^{\circ}$ 9).

\section{Les variantes}

Le schéma montre les variantes les plus courantes : en rez-de-chaussée ou à étage, les avancées présentent ou non des pignons, abritant ou non un escalier extérieur.

L'« appentis » abritant l'escalier extérieur sous lequel est aménagée une partie de la salle commune (table, bancs, éventuellement une petite resserre) ou un espace exigu servant de soue à cochon, se limite à l'aire des montagnes qui va des monts d'Arrée aux montagnes noires, soit globalement de Pleyber-Christ à Châteauneuf-du-Faou. Permettant un accès au grenier depuis l'extérieur il libère, bien sûr, l'espace intérieur de l'emprise d'un escalier, mais les raisons d'opter pour la monumentalité de celui-ci, de surcroît protégé par l'avancée du toit, ne sont pas encore clairement établies. Tout au plus on peut affirmer que l'existence même de tels escaliers signale l'importance de ces greniers commodément accessibles (fig. $\left.\mathbf{n}^{\circ} \mathbf{1 0}, \mathbf{n}^{\circ} \mathbf{1 1}\right)$. 
Figure 10

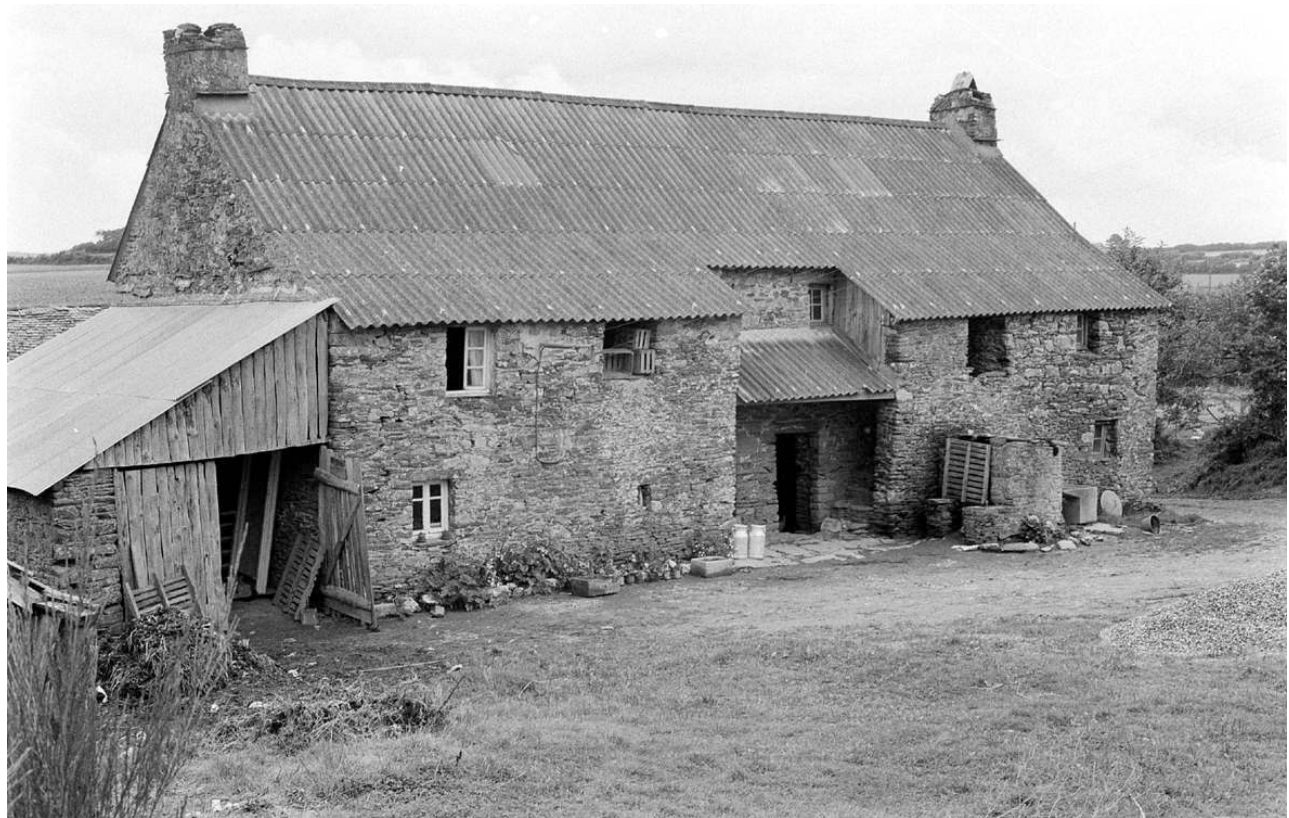

Sizun, Goas Ven. Les logis jumelés, parfaitement identiques, ont été bâtis en 1762. Chaque avancée abrite un escalier extérieur. Etat en 1970. Pape, L.

(c) Inventaire général, ADAGP, 2006.

Figure 11

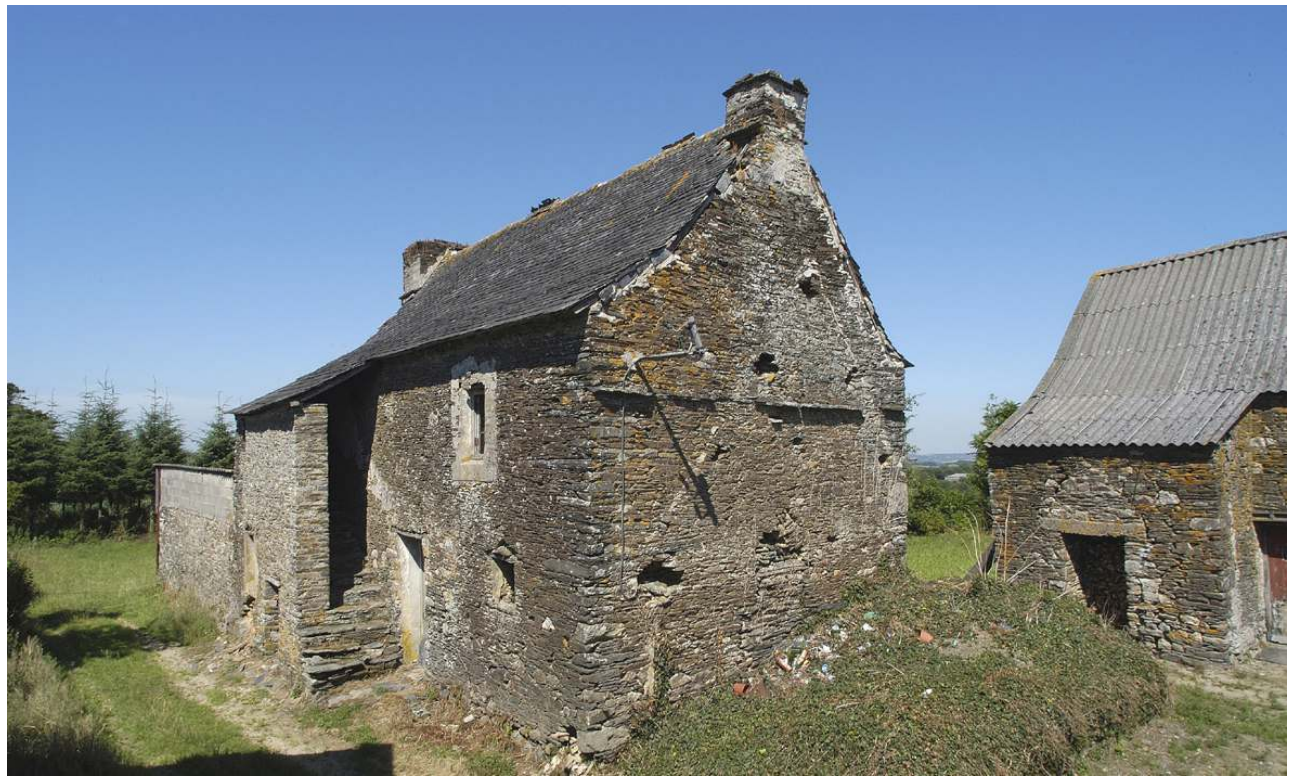

Sizun, Falzou-Baron. Cet ancien logis de ferme, à escalier de distribution extérieur, pourrait dater du milieu ou de la seconde moitié du XVIle siècle. Bègne, Bernard

(c) Inventaire général, ADAGP, 2006.

17 L'« appentis » semi-circulaire, phénomène marginal et limité au bas Léon (à l'exception des côtes), semble être un rajout tardif, bien qu'il n'augmente pas sensiblement la surface habitable. Quant à la variante, très tardive, avec deux avancées à pignon et sans escalier extérieur, elle est caractéristique du haut Léon où, suite à la première révolution agricole, 
l'habitat rural a été entièrement reconstruit à partir du milieu du XIXe siècle. On y décèle la continuité d'une tradition locale qui se transmet sans rupture, même si les volumes et l'usage de l'espace intérieur évoluent (fig. $\mathbf{n}^{\circ}$ 12).

Figure 12

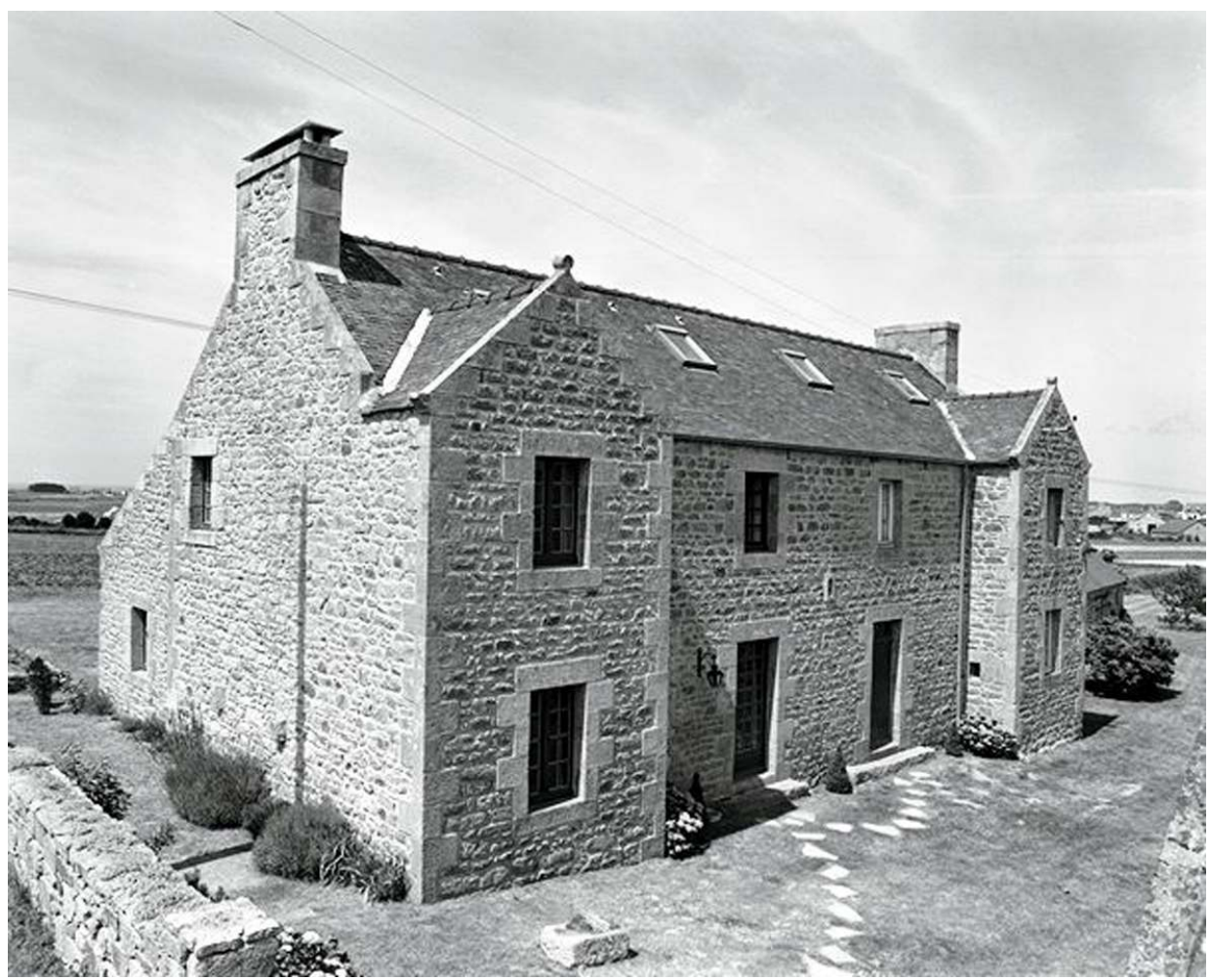

Plougoulm. Maison à double avancée caractéristique de la région de Saint-Pol-de-Léon (fin XIXe siècle). Lambart, Norbert

(c) Inventaire général, ADAGP, 1987

\section{Typo-chronologie}

Durant trois siècles, les logis à avancée sont donc caractéristiques d'une partie de l'architecture vernaculaire bretonne. Mais l'apparition et la disparition d'une variante au profit d'une autre ne sont pas aisées à établir. La coexistence de plusieurs variantes, l'absence d'une statistique monumentale suffisamment fine ou encore les remaniements intervenus au cours du temps rendent fragile toute classification trop affirmative. Il parait néanmoins possible de présenter quelques traits dominants.

Les avancées avec escalier(s) extérieur(s) de distribution existent dès la première moitié du XVIIe siècle (y en avait-il avant ?) et semblent disparaître dans la seconde moitié du XVIIIe siècle. La zone de densité de cette variante, particulièrement présente au XVIIe siècle, correspond aux monts d'Arrée (fig. $\mathbf{n}^{\circ}$ 13). 
Figure 13

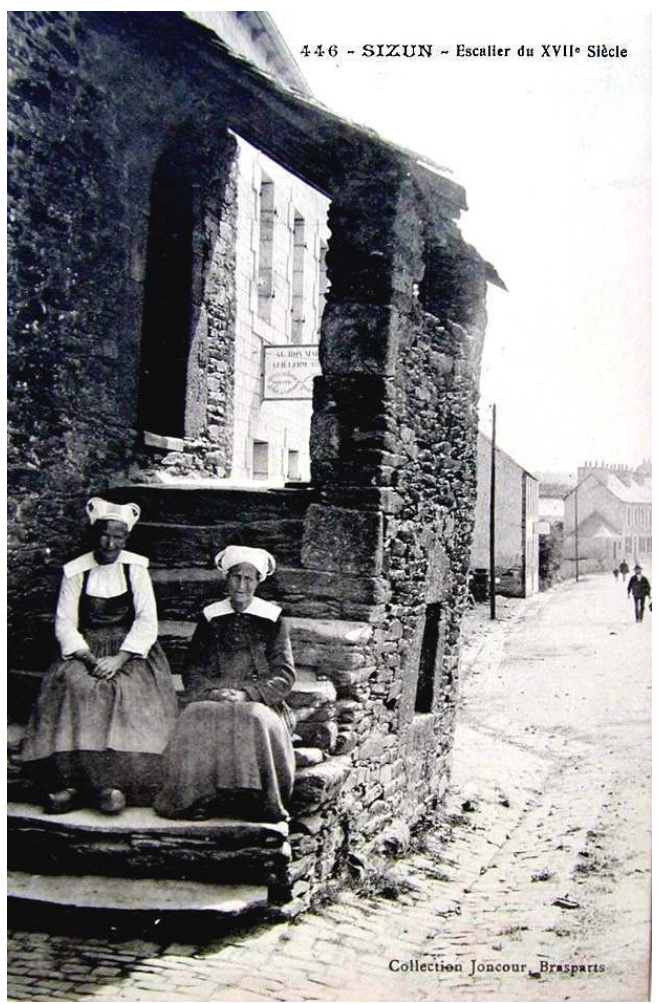

Sizun, bourg. Maison disparue. Carte postale, vers 1900 (coll. part.). Douard, Christel (c) Inventaire général, ADAGP, 2006.

20 Les avancées à pignon et étage existent, avec une grande diversité des volumes, entre la première moitié du XVIIe et le début du XXe siècle. Cette variante connaît une longévité supérieure à toutes les autres (fig. $\left.\mathbf{n}^{\circ} \mathbf{1 4}\right)$. 


\section{Figure 14}

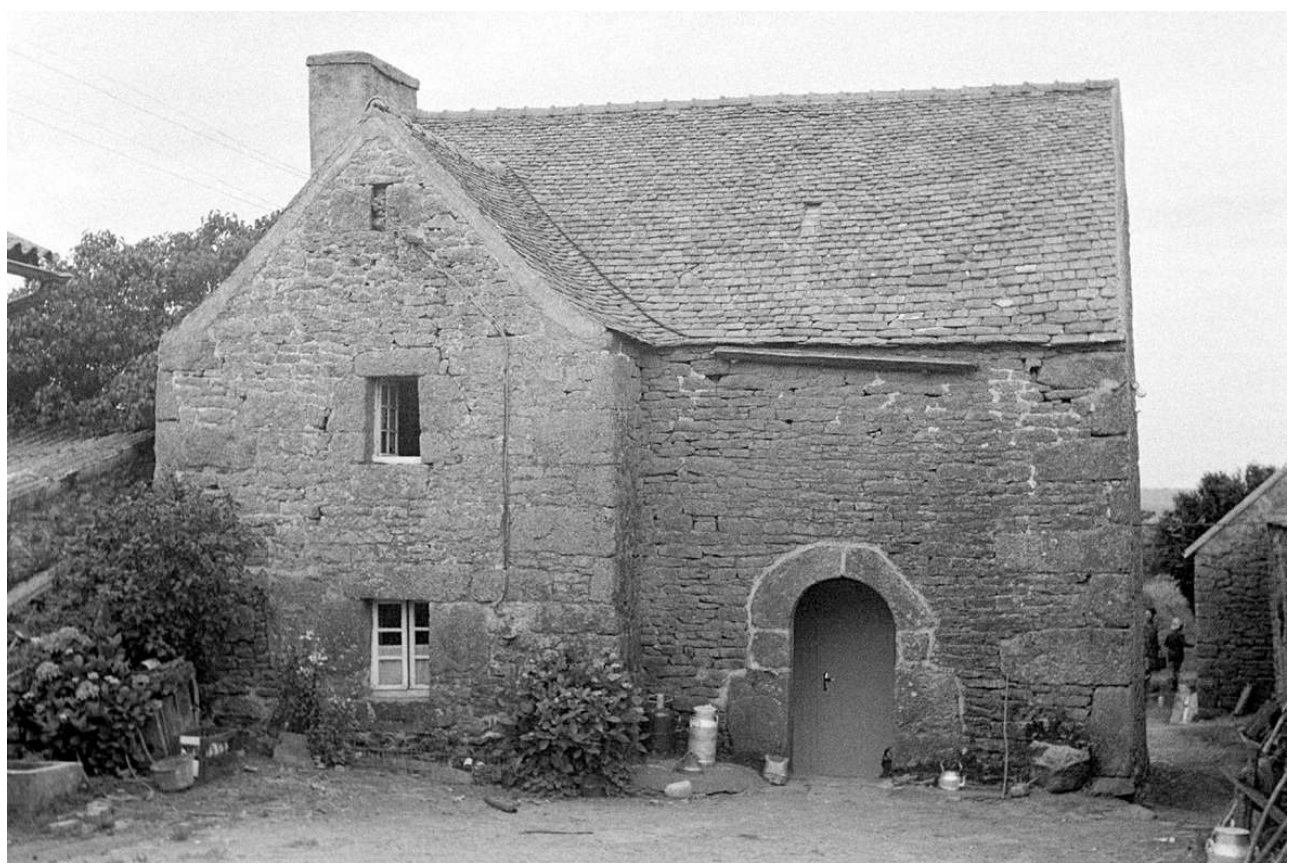

Plounéour-Ménez, Guirhoel. Logis de ferme (limite XVIle

XVIIIe siècle), état en 1969

(c) Inventaire général, ADAGP.

Les avancées sans pignon ni étage s'observent entre le début du XVIIIe siècle et le milieu, voire le 3e quart du XIXe siècle (fig. $\mathbf{n}^{\circ} \mathbf{1 5}$ ). 
Figure 15

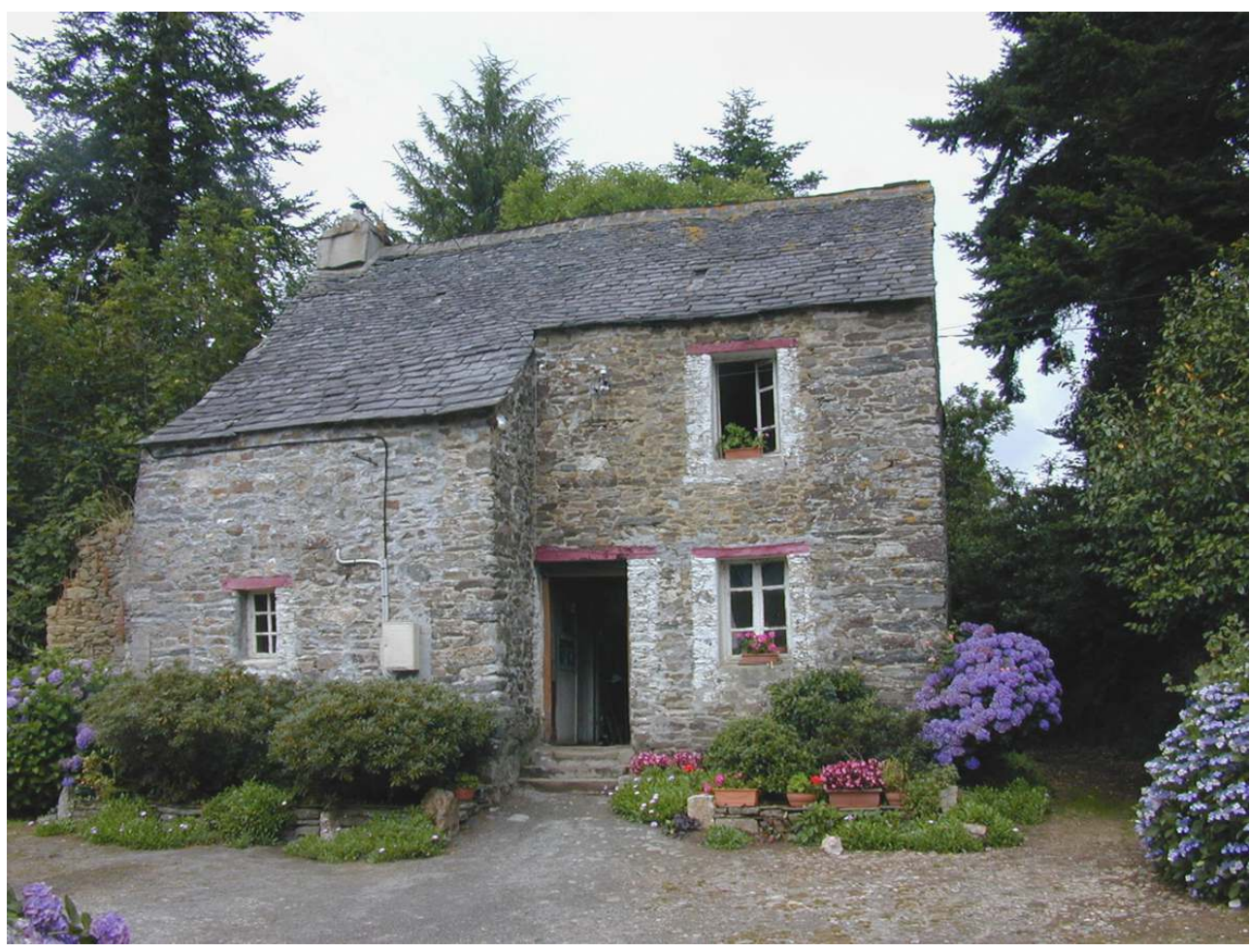

Sizun, Kerlodézan Huella. Logis daté 1833. Douard, Christel

(c) Inventaire général, ADAGP, 2006.

22 Les avancées sans pignon en rez-de-chaussée, peu répandues avant le début du XIXe siècle, ont déjà tendance à disparaître dès les années 1850. Au regard de la modestie de tels bâtiments, beaucoup ont dû disparaître. 


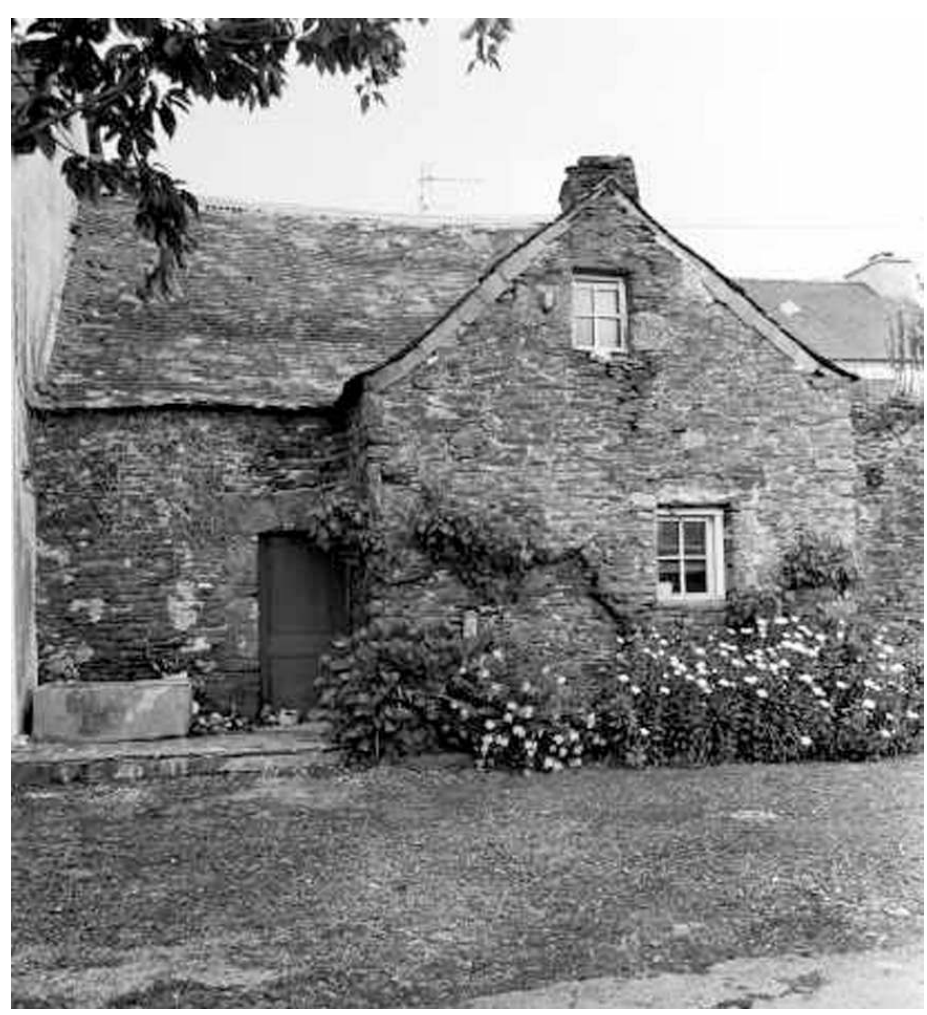

Lopérec, Bruluec. Logis daté 1833. Bègne, Bernard

(c) Inventaire général, ADAGP, 1997.

Les avancées avec pignon en rez-de-chaussée existent au moins depuis la première moitié du XIXe siècle, peut-être avant. En l'absence de chronogrammes, cette variante est difficile à dater et tend à disparaître dès le milieu du XIXe siècle (fig. $\mathbf{n}^{\circ} \mathbf{1 6}$ ).

Aujourd'hui, la disparition des témoins les plus modestes, corroborée par la surreprésentativité des témoins les plus remarquables, fait que les données dont on dispose sont à manier avec prudence. D'autant plus que dans les zones traditionnellement concernées, les édifices non remaniés tombent progressivement en ruine. A Sizun, par exemple, 25 \% des maisons à avancée répertoriées en 1970 avaient disparu en 2006.

L'érosion de ce patrimoine est donc telle que son étude in vivo ne sera bientôt plus qu'un souvenir.

\section{L'évolution vers le pseudo-régional}

$\mathrm{Au}$ début du XXe siècle, avec l'émergence du néo-régionalisme ${ }^{12}$, la maison à avancée devient un des prototypes de la maison bretonne. On la retrouve dans un premier temps, revue et corrigée par les architectes-promoteurs du néo-régionalisme, durant la période de l'entre-deux-guerres, en réponse aux commandes d'une bourgeoisie qui l'expérimente dans ses demeures balnéaires ${ }^{13}$. Les lointains échos de ce type de maison se font encore sentir après 1950 à travers les offres des entreprises du bâtiment et leurs catalogues et publicités qui visent une clientèle plus populaire ${ }^{14}$. Le modèle sera, jusqu'à nos jours, décliné jusqu'à se figer dans des formules stéréotypées, ce qui, pour André Mussat, est 
bien l'expression de cette "prodigieuse fortune du pseudo-régional, une des grandes inventions de notre siècle $»^{15}$. On est désormais loin de l'équilibre et de la fonctionnalité initiaux des modèles anciens, alors que la forme architecturale, déclinée en de multiples versions, a envahi toute la région jusqu'à en faire oublier l'origine (fig. $\left.\mathbf{n}^{\circ} \mathbf{1 7}, \mathbf{n}^{\circ} \mathbf{1 8}\right)$.

Figure 17

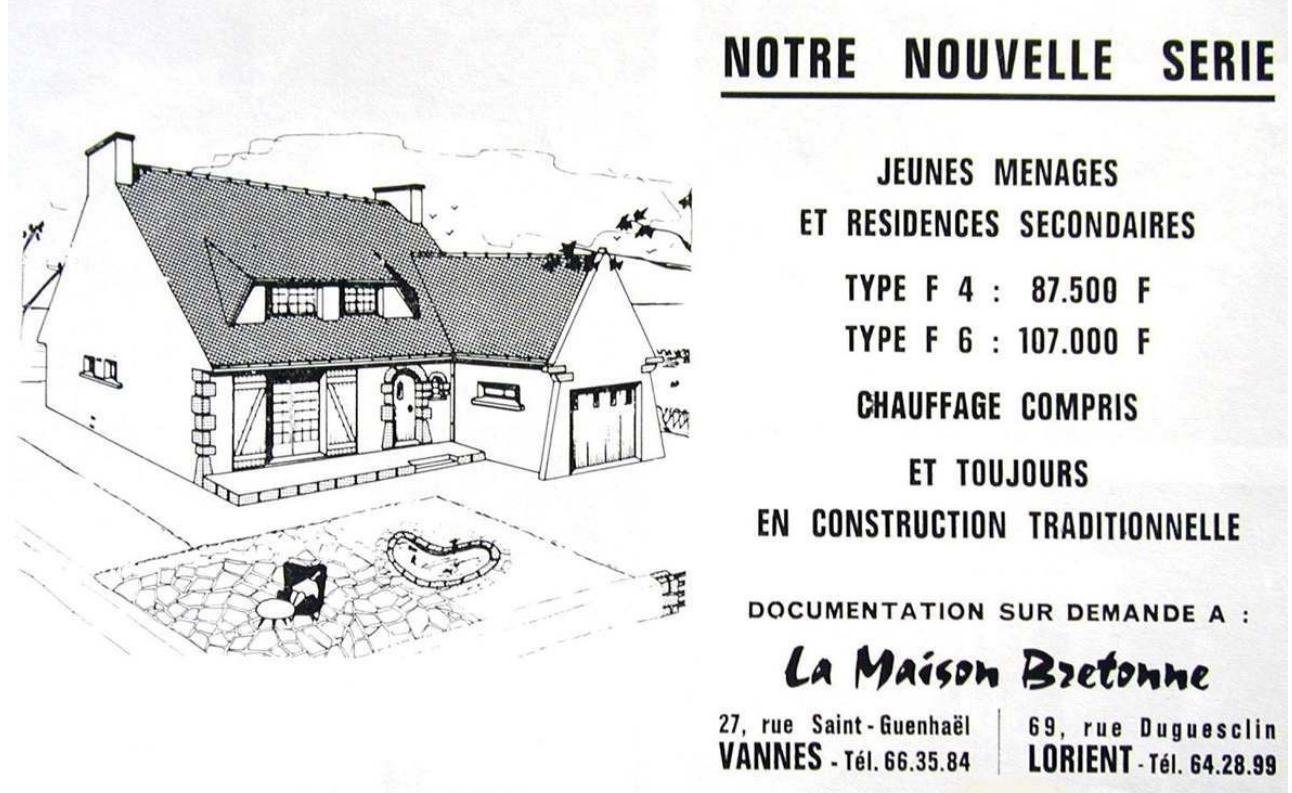

Planche extraite de la revue MAISONS DE L'OUEST, mars 1969. Douard, Christel

(c) Inventaire général, ADAGP, 2006. 


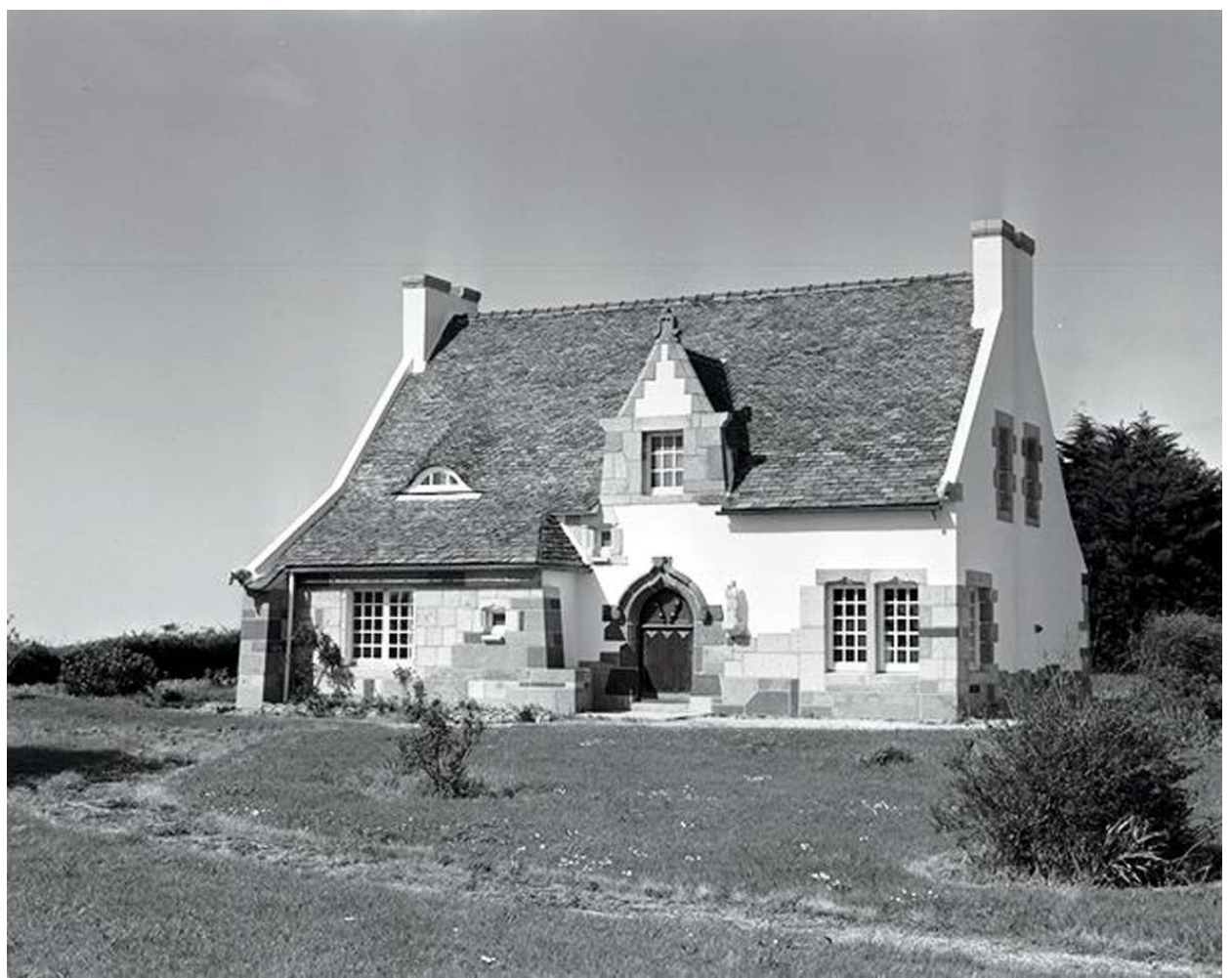

Plouénan, Valy Glas. Logis construit vers 1980. Lambart, Norbert

(C) Inventaire général, ADAGP, 1987.

Plusieurs travaux publiés sur le sujet depuis une trentaine d'années s'appuient sur certains poncifs qui, aussi bien dans le grand public qu'auprès de ceux qui habitent et aménagent ces maisons, continuent à être véhiculés sans nuance; c'est le cas de la fameuse idée reçue selon laquelle toute maison à avancée serait une maison de tisserand. Seul un recensement systématique du bâti rural dans les secteurs concernés et un croisement avec les données fournies par d'autres disciplines, et plus particulièrement l'histoire et l'archéologie, permettront - s'il en est encore temps - de confirmer, d'infirmer ou de préciser davantage la récurrence des types et des variantes dans l'espace et le temps.

\section{NOTES}

1. Le présent article se base sur les travaux suivants: L'architecture rurale française. Corpus des genres, des types et des variantes. Bretagne (dir. J.Cuisenier). Musée national des arts et traditions populaires (réd. Daniel Le Couédic, Jean-René Trochet). Editions A DIE, 1999. DOUARD, Christel, LE BRIS DU REST, Erwan, DELMOTTE, Pascale. Bretagne. Habitat rural et société (Coll. Itinéraires pédagogiques, $\mathrm{n}^{\circ} 2$ ). Ministère de la Culture (DRAC Bretagne), Ministère de l'Education nationale (Inspection Académique du Finistère). Publication accompagnée d'un cédérom. 
Rennes: CRDP, 2000. DOUARD, Christel, LE BRIS DU REST, Erwan. La maison rurale. Bretagne. Dictionnaire-guide du patrimoine (dir. J.-M. Pérouse de Montclos). Paris : Monum/Editions du Patrimoine, 2002, p. $117 . \quad \mathrm{http}$ //www4.culture.fr/patrimoines/ patrimoine_architectural_et_mobilier/sribzh/main.xsp

2. SIMON, Jean-François. Tiez. Le paysan breton et sa maison. 1 : Le Léon. Douarnenez: Editions de l'Estran, 1982. SIMON, Jean-François. Les maisons à avancée du Finistère. ArMen, n - 1, 1986, p. 20-31.

3. Voir les dossiers électroniques dans : L'inventaire du Patrimoine des communes littorales des Côtes d'Armor : http://archives.cotesdarmor.fr/asp/dossiers.asp

4. Inventaire général des Monuments et des richesses artistiques de la France. Région Bretagne. Entre mer et fleuve. Le Faou et son canton (réd. C. Douard, C. Toscer-Vogel, E. Le Bris du Rest). Rennes, 1998 (Coll. Images du Patrimoine, n 174) p. 10-11.

5. Réalisée par le service régional de l'Inventaire, une étude du patrimoine bâti sur 34 communes du Parc Naturel Régional d'Armorique (Finistère), est actuellement en cours.

6. LAGADEUC, Jehan. Catholicon seu Dictionarius britonum. Dictionnaire français-latin-breton. Tréguier, 1499 (Bibliothèque municipale Rennes).

7. ROSTRENEN, Grégoire de. Dictionnaire français-celtique, ou français-breton, nécessaire à tous ceux qui veulent apprendre à traduire le français en celtique, ou en langage breton (...). Rennes : Jacques Vatar, 1732.

8. FEUTREN, Jean (abbé). Note sur quelques maisons de la commune de Pleyber-Christ d'après les sources des archives du château de Lesquiffiou. Bulletin paroissial de Pleyber-Christ, $\mathrm{n}^{\circ} 103$, 1984, p. 22-24.

9. Extrait du procès-verbal de mesurage, prisage et estimation des droits, réparatoires, édifices et superficies de Kervent, 1806 (Archives privées, commune de Lopérec, Finistère).

10. OLIER, Ernest, OLIER, Yvonne. La maison de tisserand à porche extérieur surélevé dans le Haut-Léon au XVIIe siècle. Bulletin de la société archéologique du Finistère. Quimper, 1981, p. 289-309.

11. MUSSAT, André. L'architecture régionale. Bretagne. Architecture et identités. Textes réunis par D. Leloup. (Coll. Art et Société). Rennes : Presses Universitaires de Rennes, 1997, p. 44.

12. LE COUEDIC, Daniel. Les architectes et l'idée bretonne, 1904-1945. Rennes, Saint-Brieuc, 1995.

13. BARBEDOR, Isabelle, DELIGNON, Gaëlle, ORAIN, Véronique. La Côte d'Emeraude. La villégiature balnéaire autour de Dinard et Saint-Malo (dir. B. Toulier, F. Muel). Paris : Monum/Editions du Patrimoine, 2001 (Coll. Cahiers du Patrimoine, $\mathrm{n}^{\circ}$ 60).

14. LE COUEDIC, Daniel. La maison oripeau de l'identité. Bretagne. Art, création, société. En l'honneur de Denise Delouche (dir. J.-Y. Andrieux, M. Grivel). (Coll. Art et Société). Rennes : Presses Universitaires de Rennes, 1997, p. 211-219.

15. MUSSAT, André. L'architecture régionale. Bretagne. Architecture et identités. Textes réunis par D. Leloup. (Coll. Art et Société). Rennes : Presses Universitaires de Rennes, 1997, p. 42.

\section{RÉSUMÉS}

Caractéristiques d'un vaste secteur rural du Finistère et d'une petite frange limitrophe des Côtesd'Armor, les "maisons à avancée ", souvent datées par chronogramme, ont été bâties entre le 
début du XVIIe siècle et le début du XXe siècle. Il s'agit de cerner l'aire de diffusion, les morphologies, la terminologie (française, bretonne), l'apport du document d'archives, tout comme l'impact que le modèle a pu exercer sur l'habitat - pas nécessairement rural - du XXe siècle.

"Maisons à avancée", houses with projecting parts, are characteristic of a large rural sector of the department of Finistère and a smaller coastal strip of the Côtes-d'Armor department. The buildings, often dated by the chronograms they bear, were constructed between the early 17th century and the early 20th century. Their study in an inventory programme aimed to plot their area of diffusion, their morphologies, the French and Breton terminology concerning them, the archival information on them, and their influence as a model for dwellings, not necessarily rural ones, during the 20th century.

\section{INDEX}

Mots-clés : Apothéis, Botmeur, Brasparts, Catholicon, Grégoire de Rostrenen, Jean Lagadeuc, Le Faou, Léon, Lopérec, maison à avancée, maison de tisserand, Monts d'Arrée, Pleyber-Christ, Plouénan, Plougoulm, Plounéour-Ménez, pseudo-régional, Saint-Pol-de-Léon, Sizun, Tréduder, Trégor finistérien

Keywords : pseudo-regional style, Trégor region (Finistère), weaver's house

\section{AUTEUR}

\section{CHRISTEL DOUARD}

Ingénieur d'études. Service chargé de l'inventaire général du patrimoine culturel, Région Bretagne. christel.douard@culture.gouv.fr 